Reprod. Nutr. Dévelop., 1983, 23 (1), 129-136.

\title{
Technique permettant d'améliorer la fertilité des femelles françaises frisonnes pie noire (FFPN) en climat tropical. Influence sur l'évolution de la progestérone plasmatique
}

\author{
D. GAUTHIER
}

avec la collaboration technique de G. COULAUD et H. VARO

Station de Recherches zootechniques, I.N.R.A., Domaine Duclos, 97170 Petit-Bourg, France.

Summary. A technique for improving the fertility of French Friesian cows in a tropical climate. Effect on plasma progesterone profile.

Seasonally high temperatures in tropical regions are accepted as adversely affecting reproduction in cattle. This paper reports five experiments carried out to assess the effect of a water-spray treatment on fertility and on progesterone concentration in French Friesian cows kept under a shade structure in a tropical region (Guadeloupe). Three experiments were conducted during the hot season and two during the cold season. The spray treatment ( 3 times a day for half an hour) began at the end of a treatment to induce ovulation and continued to day 10 after insemination. A comparison of sprayed and unsprayed cows during the hot season showed that fertility was higher (53 and 13 p. 100, respectively ; $P<0.05)$ and rectal temperature lower $\left(38.8\right.$ and $39.3{ }^{\circ} \mathrm{C}$, respectively; $P<0.001)$ at 3 p.m. in sprayed cows. During the cold season, fertility and rectal temperature were equivalent in both groups.

Plasma progesterone levels were compared in sprayed and unsprayed cows in both seasons and the effect of pregnancy was studied. The progesterone curves were fitted to logistic function (from day of insemination to day 17). Multivariate analysis showed a treatment-season interaction and a pregnancy effect. During the hot season, sprayed cows had higher maximal progesterone concentration and reached a maximal rate of progesterone increase sooner than unsprayed cows $13.9 \mathrm{ng} / \mathrm{ml}$ and 7.9 days $v s 3.1 \mathrm{ng} / \mathrm{ml}$ and 8.6 days, respectively ; $P<0.05)$. Pregnant cows had a higher maximal progesterone concentration and a higher maximal rate of progesterone increase than non-pregnant cows $(4.2$ and $0.73 \mathrm{ng} / \mathrm{ml} /$ day, respectively $v s 3.0$ and $0.52 \mathrm{ng} / \mathrm{ml} / \mathrm{day}$, respectively; $\mathrm{P}<0.05)$. During the cold season, only the day of maximal increase in progesterone rate was different between sprayed and unsprayed cows $(7.6$ vs $9.3 ; \mathrm{P}<0.05)$.

\section{Introduction.}

Une diminution de la fertilité et de la concentration plasmatique de progestérone des bovins pendant la saison chaude a été souvent décrite (Ingraham, Gillette et Wagner, 1971 ; Monty et Wolff, 1974 ; Folman et al., 1979). En Guadeloupe, le nombre d'inséminations nécessaires pour obtenir une fécondation chez 
la vache FFPN, maintenue à l'ombre pendant la journée, est plus élevé pendant la saison chaude $(2,7)$ que pendant le reste de l'année 11,7 ; Heyman, résultats non publiés). Cette subfertilité, liée à la température, limite la production des animaux importés dans la zone tropicale et notamment celle des femelles laitières. $C^{\prime}$ 'est pourquoi un certain nombre de systèmes permettant une amélioration de la régulation thermique des bovins et donc une augmentation de leur production ont été proposés (Wiersma, Ray et Roubicek, 1973 ; Stott et Wiersma, 1976 ; Roman-Ponce et al., 1977 ; Johnson, 1980). Mais ces systèmes sont soit trop onéreux, soit inefficaces en Guadeloupe, car même à l'ombre, les vaches Frisonnes sont en dehors de leur zone de confort thermique (Berbigier, communication personnelle). C'est pourquoi nous avons voulu étudier un système simple permettant d'améliorer la régulation thermique de ces animaux et d'augmenter leur fertilité. De plus nous avons testé l'effet d'un tel système sur l'évolution de la concentration plasmatique de progestérone.

\section{Matériel et méthodes.}

1) Milieu et animaux. - Les expériences sont réalisées au domaine expérimental de la Station de Recherches zootechniques de I'INRA en Guadeloupe. La moyenne annuelle des précipitations est de $1280 \mathrm{~mm}$. Bien que d'importantes variations entre les années soient observées, une saison des pluies laoût à novembre, $830 \mathrm{~mm}$ ) et une période sèche (décembre à juillet, $450 \mathrm{~mm}$ ) peuvent être définies. La température maximale moyenne varie entre $27^{\circ} \mathrm{C}$ (janvier) et $32{ }^{\circ} \mathrm{C}$ (août), la minimale entre $21^{\circ} \mathrm{C}$ et $25^{\circ} \mathrm{C}$. L'hygrométrie moyenne est toujours supérieure à 70 p. 100. Pendant la journée les animaux FFPN maintenus entravés et protégés du rayonnement solaire reçoivent une alimentation à l'auge ; la nuit ils pâturent des savanes de pangola (Digitaria decumbens).

2) Protocole expérimental et dosage. - L'expérience a été répétée 5 fois (tabl. 1). Lors de chaque répétition, les femelles reçoivent le traitement d'induction de l'ovulation (Progestagène, Prostaglandine, PMSG) décrit par Chupin et Pelot (1978). A partir de l'injection de PMSG ( $\mathrm{J}$ 0) elles sont réparties en deux lots équilibrés quant à l'âge et au rang de vêlage. L'un des lots (lot douché)

TABLEAU 1

Dates et nombre de femelles lors des 5 expériences

\begin{tabular}{cccc}
\hline \multirow{2}{*}{ Numéro de l'expérience } & Dates & \multicolumn{2}{c}{ Nombre d'animaux } \\
\cline { 3 - 4 } & & Lot témoin & Lot douché \\
\hline 1 & août-septembre 1979 & 8 & 7 \\
2 & août-septembre 1980 & 7 & 8 \\
3 & novembre-décembre 1980 & 6 & 6 \\
5 & janvier-février 1981 & 6 & 6 \\
\hline
\end{tabular}


reçoit une douche d'eau à température ambiante d'une durée de $30 \min$ à 10,12 et $14 \mathrm{~h}$ de $\mathrm{J} 0$ à $\mathrm{J} 11$; l'autre lot est maintenu dans les conditions habituelles (lot témoin).

Lors des répétitions $1,2,3$ et 5 les vaches sont inséminées avec du sperme provenant d'un même éjaculat, 48 et $72 \mathrm{~h}$ après l'injection de PMSG. Pour I'expérience 4, suite à des difficultés indépendantes du protocole expérimental, les animaux ne sont ni inséminés ni saillis.

Au cours des trois premières répétitions, la température rectale des animaux est mesurée à $\mathrm{J} 5$ et $\mathrm{J} 7$ entre $8 \mathrm{~h} 30$ et 9 h 30 et entre $14 \mathrm{~h} 30$ et $15 \mathrm{~h} 30$. Pour les répétitions 4 et 5 , une prise de sang est effectuée quotidiennement dans la veine jugulaire de chacune des femelles, entre $9 \mathrm{~h}$ et $10 \mathrm{~h}$ de $\mathrm{J} 2 \mathrm{à} \mathrm{J} \mathrm{18.} \mathrm{La}$ mesure de la progestérone plasmatique de ces échantillons est effectuée à l'aide du dosage radioimmunologique décrit par Yenikoye et al. (1981). Dans nos conditions la sensibilité du dosage est de $0,04 \mathrm{ng} / \mathrm{ml}$. La précision intra-dosage, exprimée par les coefficients de variation, est respectivement de 8 et de $13 \mathrm{p}$. 100 pour des concentrations de 4 et $10 \mathrm{ng} / \mathrm{ml}$ (9 répétitions par concentration). Les coefficients inter-dosages sont respectivement de 10 et de 20 p. 100 pour ces deux concentrations.

3) Méthodes d'analyse statistique. - L'évolution de la progestérone a pu être ajustée à une courbe de type logistique se résumant à 3 paramètres ayant une signification biologique (Yenikoye et al., 1981): $\mathrm{M}=$ niveau maximum de progestérone, $\mathrm{Tm}=$ moment où le taux d'accroissement de la progestérone est maximal, $\mathrm{Pm}=$ taux d'accroissement de la progestérone à l'instant Tm (fig. 1). Les paramètres sont estimés par la méthode des moindres carrés pour chacune des femelles.

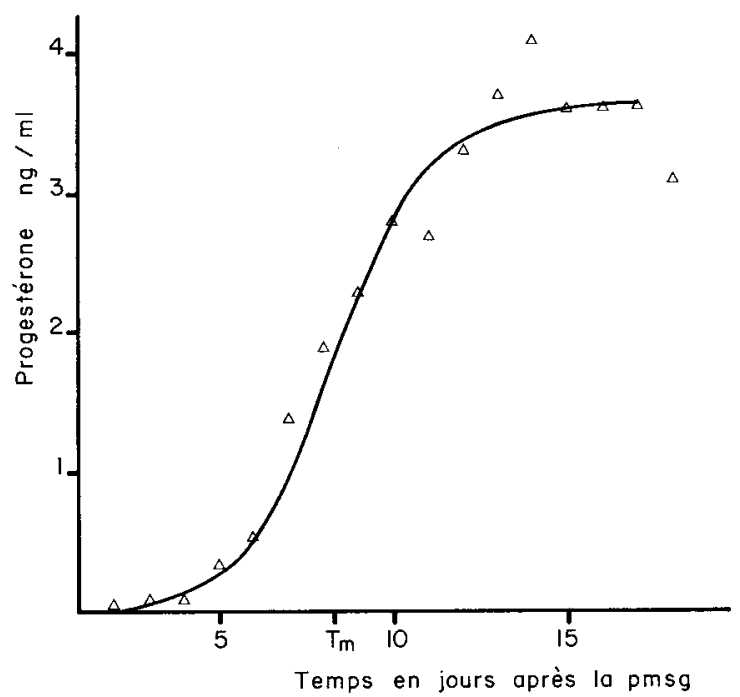

FIG. 1. - Evolution moyenne de la progestérone plasmatique chez la vache FFPN en Guadeloupe après un traitement d'induction de l'ovulation. $\Delta$ : Valeurs mesurées; --_- : courbe ajustée. 
Les effets des facteurs aspersion et saison (répétition 4 et 5) ou aspersion et état physiologique (répétition 5) sont analysés sur l'ensemble de ces trois variables à l'aide du modèle d'analyse de variance multivariée suivant (Manova).

$Y_{\mathrm{ijl}}=\mu+\alpha_{\mathrm{i}}+\beta_{\mathrm{j}}+(\alpha \beta)_{\mathrm{ij}}+\epsilon_{\mathrm{ijl}}$

où $Y_{\text {iil }}=1$-ième individu du i-ième niveau du 1 er facteur et $j$-ième niveau du deuxième $\left(\mathrm{Y}_{\mathrm{ij} \mid}\right.$ représente donc une courbe d'évolution de la progestérone $; \mu=$ moyenne de la population; $\alpha_{\mathrm{i}}=$ effet du 1 er facteur au niveau $\mathrm{i} ; \beta_{\mathrm{j}}=$ effet $\mathrm{du}$ $2^{\mathrm{e}}$ facteur au niveau $\mathrm{j} ;(\alpha \beta)_{\mathrm{ij}}=$ effet de l'interaction du $1^{\text {er }}$ et du $2^{\mathrm{e}}$ facteur aux niveaux $\mathrm{i}$ et $\mathrm{j} ; \varepsilon_{\mathrm{ijl}}=$ résidus de l'individu $\mathrm{Y}_{\mathrm{ij}}$ et où les quantités $Y, \mu, \alpha, \beta(\alpha \beta)$ et $\epsilon$ sont des matrices 3 lignes.

L'interaction et les effets principaux sont alors testés par la statistique de Bartlett appliquée au rapport $\frac{|W|}{|B+W|}$ où $|W|$ désigne le déterminant de la matrice des covariances intra-population et $|B+W|$ le déterminant de la somme des matrices de covariance intra- et inter-populations (Dagnelie, 1977). Lorsque l'interaction est significative le modèle devient $Y_{i j l}=\mu+\alpha_{i j}+\epsilon_{i j l}$, où $\alpha_{i j}$ est un facteur fictif combiné à partir des deux premiers ; si l'interaction n'est pas significative l'additivité des deux facteurs est retenue.

De plus l'influence de l'aspersion, de l'état physiologique et de la saison sur chaque variable séparément est étudiée à l'aide de l'analyse de variance univariée (Snedecor et Cochran, 1971).

Par ailleurs, les différences de température rectale et de fertilité sont étudiées respectivement par les tests de Student et de $\chi^{2}$.

\section{Résultats.}

Les températures moyennes de l'air, minimales et maximales, pendant la durée des expériences, mesurées sous abri sont respectivement de $25,3{ }^{\circ} \mathrm{C}$ et $29,5^{\circ} \mathrm{C}$ en septembre (expériences $1,2,5$ ), de $23^{\circ} \mathrm{C}$ et $28^{\circ} \mathrm{C}$ en novembre (expérience 3 ), $21,5^{\circ} \mathrm{C}$ et $28,5^{\circ} \mathrm{C}$ en janvier (expérience 4 ).

1) Fertilité (tabl. 2). - Au mois de septembre, la fertilité des femelles douchées est supérieure à celle des témoins (53 vs 13 p. $100 ; P<0,05)$. Au mois de novembre la fertilité des deux lots est identique (33 p. 100).

TABLEAU 2

Fertilité (p. 100) à /'cestrus induit de femelles douchées ou non

\begin{tabular}{|c|c|c|c|}
\hline Mois & Août-septembre & Novembre-décembre & Niveau de signification \\
\hline Lot témoin & 13 & $33(6)$ & N.S. \\
\hline Lot douché & $53(15)$ & $33(6)$ & N.S. \\
\hline Niveau de signification & $P<0,05$ & N.S. & \\
\hline
\end{tabular}

$(1)=$ Nombre de femelles par lot

fertilité $=\frac{\text { nombre de femelles mettant bas }}{\text { nombre de femelles inséminées }} \times 100$. 
2) Température rectale (tabl. 3). - Les températures rectales des animaux, mesurées le matin à $9 \mathrm{~h}$ au mois de septembre, sont identiques d'un lot à l'autre $\left(38,7^{\circ} \mathrm{C}\right)$.

TABLEAU 3

Température rectale moyenne des femelles douchées ou non à deux saisons $(\mathrm{m} \pm \sigma$ )

\begin{tabular}{ccccc}
\hline \multirow{2}{*}{ Lots } & \multicolumn{2}{c}{ Août-septembre } & \multicolumn{2}{c}{ Novembre-décembre } \\
\cline { 2 - 5 } & $9 \mathrm{~h}$ & $15 \mathrm{~h}$ & $9 \mathrm{~h}$ & $15 \mathrm{~h}$ \\
\hline Témoin & $38^{\circ} 7^{\mathrm{a}} \pm 0,1$ & $39^{\circ} 3^{\mathrm{b}} \pm 0,4$ & $38^{\circ} 4^{\mathrm{a}} \pm 0,2$ & $38^{\circ} 5^{\mathrm{a}} \pm 0,2$ \\
\hline Douché & $38^{\circ} 7^{\mathrm{a}} \pm 0,2$ & $38^{\circ} 8^{\mathrm{a}} \pm 0,2$ & $38^{\circ} 4^{\mathrm{a}} \pm 0,3$ & $38^{\circ} 7^{\mathrm{a}} \pm 0,3$ \\
\hline
\end{tabular}

Les valeurs avec des lettres identiques ne sont pas statistiquement différentes.

A $15 \mathrm{~h}$, en septembre, les femelles du lot témoin ont une température rectale $\left(39,3^{\circ} \mathrm{C}\right)$ supérieure à celle observée en novembre $\left(38,5^{\circ} \mathrm{C} ; \mathrm{P}<0,01\right)$ et la température des femelles du lot douché est inférieure à celle des témoins $\left(38,8^{\circ} \mathrm{C} ; \mathrm{P}<0,01\right)$; cette différence $\mathrm{n}^{\prime}$ apparaît plus en novembre $\left(38,7^{\circ} \mathrm{C}\right)$.

II existe donc une interaction entre la saison et le traitement sur la température rectale.

3) Evolution de la progestérone. - Quels que soient les individus, les traitements et les saisons, le pourcentage de variance totale de l'évolution de la progestérone expliquée par le modèle est toujours supérieur à 91 .

La concentration ajustée moyenne de progestérone, très faible à $J 2$ $(0,01 \mathrm{ng} / \mathrm{ml}$, environ), augmente dès $\mathrm{J} 4 \mathrm{avec}$ un taux d'accroissement maximum entre $J 8$ et $J 9(0,65 \mathrm{ng} / \mathrm{ml} /$ jour $)$ et atteint entre $J 13$ et $J 14$, sa valeur maximale : $3,6 \mathrm{ng} / \mathrm{ml}$ (fig. 1 ).

\section{TABLEAU 4}

Valeurs des paramètres définissant l'évolution de la progestérone plasmatique au cours de la phase lutéale $(\mathrm{m} \pm \sigma)$

\begin{tabular}{lcccccccc}
\hline \multicolumn{1}{c}{ Mois } & \multicolumn{4}{c}{ Août-septembre } & & Janvier-février \\
\hline Paramètres & $M$ & Tm & Pm & $\begin{array}{c}\text { nombre de } \\
\text { femelles }\end{array}$ & M & Tm & Pm & $\begin{array}{c}\text { nombre de } \\
\text { femelles }\end{array}$ \\
\hline Lot témoin & $3,1 \pm 0,7$ & $8,6 \pm 1,0$ & $0,65 \pm 0,24$ & 6 & $3,7 \pm 1,5$ & $9,3 \pm 1,7$ & $0,61 \pm 0,20$ & 6 \\
\hline Lot douché & $3,9 \pm 0,8$ & $7,9 \pm 0,6$ & $0,62 \pm 0,24$ & 7 & $3,6 \pm 0,4$ & $7,6 \pm 0,3$ & $0,70 \pm 0,40$ & 6 \\
\hline $\begin{array}{l}\text { Niveau de } \\
\text { signification }\end{array}$ & $p<0,05$ & $p<0,05$ & N.S. & & N.S. & $p<0,05$ & N.S.
\end{tabular}

$\mathrm{M}=$ concentration maximum de progestérone $(\mathrm{ng} / \mathrm{ml}) . \mathrm{Tm}=$ temps au maximum du taux de sécrétion (jours après la PMSG). $\mathrm{Pm}=$ taux de sécrétion maximum $(\mathrm{ng} / \mathrm{ml} / \mathrm{jour})$. 
L'analyse de variance multivariée appliquée aux 3 paramètres estimés (niveau maximum, temps au maximum du taux d'accroissement et valeur de ce taux) permet de conclure qu'il existe une interaction entre l'effet de la saison et celui de l'aspersion; de plus l'effet de l'aspersion est significatif, celui de la saison ne l'est pas. Le même test appliqué aux données obtenues lors de la répétition de saison chaude (septembre), seule saison où les inséminations sont effectuées, montre un effet significatif de l'arrosage et de l'état physiologique (gravide ou non), l'interaction ne l'étant pas.

a) Etude de l'interaction saison-aspersion (tabl. 4). - Le niveau maximum de progestérone est plus élevé, en septembre, chez les vaches douchées $(3,9 \mathrm{ng} / \mathrm{ml})$ que chez les témoins $(3,1 \mathrm{ng} / \mathrm{ml} ; \mathrm{p}<0,05)$, alors qu'en janvier il n'est pas différent. En saison fraîche ce niveau semble plus élevé qu'en saison chaude bien que la différence ne soit pas significative.

Le taux maximum de sécrétion de progestérone est atteint plus rapidement, en janvier et en septembre, par les femelles douchées (respectivement 7,6 et 7,9 jours) que par celles de l'autre lot $(9,3$ et 8,6 jours ; $p<0,05)$, mais il ne diffère ni entre les lots ni entre les saisons.

b) Etude de l'influence de l'état physiologique (tabl. 5). - En septembre, les femelles gravides après insémination artificielle ont un niveau de progestérone et un taux de sécrétion maximale plus élevés que ceux des femelles non gravides (respectivement $4,2 \mathrm{ng} / \mathrm{ml}$ et $0,73 \mathrm{ng} / \mathrm{ml} /$ jour $v s 3,0 \mathrm{ng} / \mathrm{ml}$ et $0,52 \mathrm{ng} / \mathrm{ml} /$ jour ; $p<0,05)$, celui-ci étant atteint simultanément dans les deux lots.

TABLEAU 5

Valeurs des paramètres définissant l'évolution de la progestérone plasmatique au cours de la phase lutéale ; influence de l'état physiologique $(m \pm \sigma)$

\begin{tabular}{cccc}
\hline Paramètres & $\mathrm{M}$ & $\mathrm{Tm}$ & $\mathrm{Pm}$ \\
\hline $\begin{array}{c}\text { Femelles non gravides } \\
(7)\end{array}$ & $3,0 \pm 0,8$ & $8,4 \pm 0,8$ & $0,52 \pm 0,20$ \\
\hline $\begin{array}{c}\text { Femelles gravides } \\
(6)\end{array}$ & $4,2 \pm 0,7$ & $8,0 \pm 0,9$ & $0,73 \pm 0,26$ \\
\hline Niveau de signification & $\mathrm{p}<0,05$ & N.S. & $\mathrm{p}<0,05$ \\
\hline
\end{tabular}

$\mathrm{M}$ : niveau maximum de progestérone $(\mathrm{ng} / \mathrm{ml})$. Tm : temps au maximum du taux de sécrétion (jours après la PMSG). Pm : taux de sécrétion maximum (ng/ml/jour). ( ) : nombre de femelles.

\section{Discussion.}

Notre travail confirme que pendant la saison chaude et humide la température rectale des femelles FFPN est élevée et leur fertilité faible. Il montre que, même en zone tropicale humide, l'aspersion des animaux laitiers améliore leur régulation thermique, et que réalisée à des moments adéquats par rapport à 
l'œstrus et à l'ovulation, elle entraîne une augmentation du taux de gestation. En zone chaude et sèche cette technique est déjà utilisée (Johnson, 1980) pour aider les animaux à minimiser les effets du stress climatique.

En effet, une température rectale élevée dans les heures qui suivent l'insémination artificielle, est toujours associée à une subfertilité (Ulberg et Burfening, 1967 ; Dunlap et Vincent, 1971; Gwazdauskas, Thatcher et Wilcox, 1973 ; Thatcher, 1974). II en est de même lorsque le stress thermique a lieu au début de la gestation (Dunlap et Vincent, 1971 ; Stott et Wiersma, 1973 ; Ortavant et Loir, 1978 ; Sawyer, 1979). En Floride, une simple structure protégeant les femelles Holstein du rayonnement solaire permet de diminuer significativement leur température rectale et d'augmenter leur fertilité, de façon peu onéreuse, pendant la saison chaude (Roman-Ponce et al., 1977). Mais, dans notre contexte d'élevage, avec des températures de l'air plus élevées, une telle structure ne suffit pas puisque la fertilité de femelles FFPN dans ces conditions n'est en moyenne que de 13 p. 100 pendant la saison chaude. La mise en chambre climatisée depuis le jour de l'œstrus jusqu'au $6^{e}$ jour post insémination (Stott et Wiersma, 1976), la ventilation continue des bâtiments pendant la journée (Folman et al., 1979), l'évaporation intense d'eau dans l'étable, l'arrosage du toit (Johnson, 1980) améliorent la régulation thermique des femelles et augmentent également leur fertilité. Mais toutes ces techniques sont coûteuses par rapport à celle que nous utilisons.

La valeur maximale ajustée moyenne de progestérone $(3,6 \mathrm{ng} / \mathrm{ml})$ et le taux maximum d'accroissement $(0,65 \mathrm{ng} / \mathrm{ml} /$ jour $)$ sont inférieurs à ceux observés chez la génisse FFPN en climat tempéré $(6,6 \mathrm{ng} / \mathrm{ml}$ et $1,3 \mathrm{ng} / \mathrm{ml} /$ jour ; Yenikoye et al., 1981), mais identiques à ceux obtenus en zone chaude et humide (RomanPonce, Thatcher et Wilcox, 1981). L'environnement tropical semble donc altérer la sécrétion de progestérone des bovins de type FFPN. Cette action peut être due à la température élevée qui diminue la sécrétion de LH (Madan et Johnson, 1973 ; Miller et Alliston, 1974).

Dans notre expérience, la fréquence des prélèvements sanguins et la puissance de l'analyse statistique permettent de montrer clairement l'influence de I'aspersion sur l'augmentation de la progestérone plasmatique pendant la saison chaude, ce qui avait déjà été suggéré par Stott et Wiersma (1973). Cette augmentation pourrait s'expliquer également par une action au niveau des hormones gonadotropes.

II est connu que la présence d'un embryon entraîne une augmentation de la progestérone dès le $10^{\mathrm{e}}$ jour post insémination (Henricks, Dickey et Niswender, 1970 ; Hansel, 1981). En revanche des relations, entre la sécrétion de progestérone et la présence d'un embryon viable, établies dès le $7^{e}$ jour (différence de taux de sécrétion) n'avaient pas encore été signalées.

Ces résultats permettent de penser que la plus faible fertilité des femelles témoins peut ainsi être due à une évolution inadéquate de la progestérone ; toutefois l'action directe de la température sur la survie embryonnaire (Ortavant et Loir, 1978) n'a pas été testée au cours de ces expériences. 


\section{Références}

CHUPIN D., PELOT J., 1978. Fertility of dairy cows treated with implants, prostaglandin analog and PMSG. Theriogenology, 10, 307-311.

DAGNeLIE P., 1977. Théorie et méthodes statistiques, Vol. 3. Ed. J. Ducolot, S. A. Gembloux.

DUNLAP S. E., VINCENT C. K., 1971. Influence of postbreeding thermal stress on conception rate in beef cattle. J. anim. Sci., 32, 1216-1218.

FOLMAN Y., BERMAN A., HERZ Z., KAIM M., ROSENBERG M., MAMEN M., GORDIN S., 1979. Milk yield and fertility of high-yielding dairy cows in a sub-tropical climate during summer and winter. J. Dairy Res., 46, 411-425.

GWAZDAUSKAS F. C., THATCHER W. W., WILCOX C. J., 1973. Physiological, environmental and hormonal factors at insemination which may affect conception. J. Dairy Sci., 56, 873-877.

HANSEL W., 1981. Plasma hormone concentrations associated with early embryo mortality in heifers. J. Reprod. Fert., Suppl. 30, 231-239.

HENRICKS D. M., DICKEY J. F,, NISWENDER G. D., 1970. Serum luteinizing hormone and plasma progesterone levels during the estrous cycle and early pregnancy in cows. Biol. Reprod., 2, 346-351.

INGRAHAM R. H., GILLETTE D. D., WAGNER W. D., 1971. Relationship of temperature and humidity to conception rate of Holstein cows in subtropical climate. J. Dairy Sci., 57, 476-481.

JOHNSON H. D., 1980. Environmental management of cattle to minimize the stress of climatic change. Biometeorology, 7, 65-78.

MADAN M. L., JOHNSON H. D., 1973. Environmental heat effects on bovine luteinizing hormone. J. Dairy Sci., 56, 1420-1423.

MILLER H. L., ALLISTON C. W., 1974. Influence of programmed circadian temperature changes upon levels on luteinizing hormone in the bovine. Biol. Reprod., 11, 187-190.

MONTY D. E., WOLFF L. K., 1974. Summer heat stress and reduced fertility in Holstein-Friesian cows in Arizona. Am. J. vet. Res., 35, 1495-1500.

ORTAVANT R., LOIR M., 1978. The environment as a factor in reproduction in farm animals. 4th World Conf. anim. Product., Buenos-Aires, 1.

ROMAN-PONCE H., THATCHER W. W., BUFFINGTON D. E., WILCOX C. J., VAN HORN H. H., 1977. Physiological and production responses of dairy cattle to a shade structure in a subtropical environment. J. Dairy Sci., 60, 424-430.

ROMAN-PONCE H., THATCHER W. W., WILCOX C. J., 1981. Hormonal interrelationships and physiological responses of lactating dairy cows to a shade management system in a subtropical environment. Theriogenology, 16, 139-154.

SAWYER G. T., 1979. The influence of radiant heat load on reproduction in the Merino ewe. I. The effect of timing and duration of heating. Aust. J. agric. Res., 30, 1133-1141.

SNEDECOR G. W., COCHRAN W. G., 1971. Statistical methods. 6th ed. Ed. lowa State Univ. Press.

STOTT G. H., WIERSMA F., 1973. Climatic thermal stress, a cause of hormonal depression and low fertility in bovine. Int. J. Biometeorol., 17, 115-122.

STOTT G. H., WIERSMA F., 1976. Short term thermal relief for improved fertility in dairy cattle during hot weather. int. J. Biometeorol., 20, 344-350.

THATCHER W. W., 1974. Effects of season, climate and temperature on reproduction and lactation. J. Dairy Sci, 57, 360-368.

ULBERG L. C., BURFENING P. J., 1967. Embryo death resulting from adverse environment on spermatozoa or ova. J. anim. Sci, 26, 571-577.

WIERSMA F., RAY D., ROUBICEK C., 1973. Modified environment for beef in hot climates. Transactions of A.S.A.E., 16, 348-353.

YENIKOYE A., MARIANA J. C., LEY J. P., JOLIVET E., TEROUI M., LEMON-RESPLANDY M., 1981. Modèle mathématique de l'évolution de progestérone chez la vache : application et mise en évidence de différences entre races. Reprod. Nutr. Dévelop., 21, 561-575. 